\title{
RGD Mutation of the Heparin Binding II Fragment of Fibronectin for Guiding Mesenchymal Stem Cell Behaviour on Titanium Surfaces
}

\author{
Jordi Guillem-Marti a,b,1, Maria Gelabert a,b, Aina Heras-Parets a,b, Marta Pegueroles a,b, \\ Maria-Pau Ginebra ${ }^{\mathrm{a}, \mathrm{b}, \mathrm{c}}$, Jose Maria Manero a,b
}

${ }^{a}$ Biomaterials, Biomechanics and Tissue Engineering Group, Department of Materials Science and Metallurgical Engineering, Universitat Politècnica de Catalunya (UPC), 08930 Barcelona, Spain

${ }^{\mathrm{b} B a r c e l o n a}$ Research Center in Multiscale Science and Engineering, Universitat Politècnica de Catalunya, 08930 Barcelona, Spain

${ }^{\mathrm{c}}$ Institute for Bioengineering of Catalonia (IBEC), Barcelona Institute of Science and Technology (BIST), 08028 Barcelona, Spain

${ }^{1}$ Corresponding author:

Jordi Guillem-Marti

Biomaterials, Biomechanics and Tissue Engineering group

Department of Materials Science and Metallurgical Engineering

Universitat Politècnica de Catalunya

Avinguda Eduard Maristany 10-14

08930 Barcelona, Spain

Phone number: 0034934137218

jordi.guillem.marti@upc.edu

Keywords: Recombinant protein, Fibronectin, Mutation, Growth Factor, Titanium, Osseointegration 


\begin{abstract}
Installing bioactivity on metallic biomaterials by mimicking the extracellular matrix (ECM) is crucial for stimulating specific cellular responses to ultimately promote tissue regeneration. Fibronectin is an ECM protein commonly used for biomaterial functionalization. The use of fibronectin recombinant fragments is an attracting alternative to the use of full-length fibronectin due to the relatively low cost and facility of purification. However, it is necessary to combine more than one fragment, e.g. the cell attachment site (CAS) and the heparin binding II (HBII), either mixed or in one molecule to obtain complete activity. In the present study, we proposed to install adhesion capacity to the HBII fragment by an RGD gain-of-function DNA mutation retaining its cell differentiation capacity thereby producing a small and very active protein fragment. The novel molecule, covalently immobilized onto titanium surfaces maintained the growth factor binding capacity and stimulated cell spreading, osteoblastic cell differentiation and mineralization of human mesenchymal stems cells compared to the HBII native protein. These results highlight the potential capacity of gain-of-function DNA mutations in the designing of novel molecules for the improvement of osseointegration properties of metallic implant surfaces.
\end{abstract}




\section{INTRODUCTION}

Titanium (Ti) is the most widely used biomaterial for bone tissue implantation due to its good mechanical properties and biocompatibility. ${ }^{1}$ However, in some circumstances the stability of metallic implants is compromised due to reduced osseointegration at the implant-tissue interface mainly attributed to the lack of bioactivity. To overcome this limitation, recent studies are focused on the functionalization of Ti surface mimicking the natural environment of cells, ${ }^{2,3}$ this is, the extracellular matrix (ECM). The ECM is composed of several molecules secreted by the cells that, in addition to providing structural and mechanical support for tissues and interacting with cells through some cell-surface receptors like integrins, is able to bind soluble molecules present in the extracellular fluids like the growth factors, ${ }^{4}$ which are known to play a crucial role in tissue regeneration.

In this context, there is a growing interest in designing functionalized biomaterials that incorporate ECM growth factor-binding domains. This has clear advantages compared to the use of growth factor-based biomaterials in tissue engineering. ${ }^{5-7}$ This is because in the latter strategy undesired side effects difficult their clinical application ${ }^{8,9}$ whereas in the former strategy spatial and temporal controlled release of growth factors may drive tangible therapeutic effects. ${ }^{10}$

Among the different ECM proteins, fibronectin is one of the most well-known. It contains several domains that interact not only with cells but also with other proteins, mediating many cellular processes such as cell adhesion, migration, growth and differentiation. ${ }^{11}$ Although the use of full-length fibronectin for functionalizing Ti implants has demonstrated improved osteoconduction capacity, ${ }^{12}$ its use for clinical applications is hampered because only small fibronectin quantities can be purified from human plasma ${ }^{13}$ and full-length proteins are sensitive to proteolytic degradation and 
may induce immune responses. ${ }^{14}$ An alternative strategy is the use of fibronectin protein fragments obtained by DNA recombinant methodologies that can be engineered containing active domains in small molecules that retain functionalities. In vitro and in vivo use of fibronectin fragments has shown much more potency than full-length fibronectin. ${ }^{15}$ In this regard, the cell attachment site (CAS) recombinant fragment from fibronectin, which encompasses at least the type $\mathrm{III}_{10}$ domain (which contains the cell adhesive RGD motif) and the type $\mathrm{III}_{9}$ domain (which contains the PHSRN synergistic sequence) has proved enhanced osseoinduction and osseintegration capacities when functionalized on Ti implants. ${ }^{16,17}$

Recent studies however have evidenced that the presence of heparin binding II (HBII) recombinant fragment from fibronectin is necessary to achieve complete activity when using CAS-functionalized surfaces. ${ }^{18,19}$ The HBII fragment acts as a highly promiscuous growth factor binding domain, ${ }^{20}$ allowing strong crosstalk between integrins and growth factor receptors when immobilized close to the CAS fragment. ${ }^{21}$ Thus, the combination of both fragments promotes higher osseoinduction compared to each fragment alone ${ }^{19}$. In addition, HBII fragment also plays a pivotal role in cell adhesion thorough the binding to Syndecan-4 cell surface heparan sulphate proteoglycan. ${ }^{22}$ This interaction induces focal adhesion formation and maturation only when interaction of CAS with integrins is present. ${ }^{23}$

Nonetheless, the simultaneous immobilization of both CAS and HBII fragments does not guarantee reproducibility of results because the percentage of each fragment at the surface cannot be controlled, due to adsorption competition. ${ }^{19}$ Alternatively, the generation of a single molecule containing both CAS and HBII fragments generates a bigger protein, which is more difficult to obtain and may be less stable after immobilization. 
To overcome these limitations, the aim of the present study was to functionalize the $\mathrm{Ti}$ surface with a single recombinant protein able to promote both adhesion and differentiation activities, in view to improve implant osseointegration. To achieve this objective we proposed to introduce a mutation in the HBII DNA sequence in order to include an RGD sequence that is expected to promote cell adhesion while retaining its cell differentiation properties. After the mutation, the growth factor binding capacity was checked measuring the interaction of the functionalized surfaces with BMP-2 and TGF- $\beta 1$. Cellular activities of the novel protein covalently attached to Ti were evaluated in terms of cell adhesion, cell spreading, cell proliferation, cell differentiation and mineralization using human mesenchymal stem cells (hMSCs) in order to elucidate the osseoinduction capacity.

\section{MATERIALS AND METHODS}

\subsection{Titanium Samples}

Commercially pure Ti grade 2 discs of $10 \mathrm{~mm}$ diameter and $2 \mathrm{~mm}$ thickness were grinded with silicon carbide papers of grit 320, 800, 1200 and 2500 (Struers, Spain). Then, the discs were mirror polished with colloidal silica $(0.05 \mu \mathrm{m}$ particle size $)$ and ultrasonically cleaned with cyclohexane, isopropanol, deionized water, ethanol and acetone ( 3 x 5 min each).

\subsection{Synthesis of Recombinant Fibronectin Fragments}

Human fibronectin fragments CAS, spanning the 8-10th type III repeats, and HBII, spanning the 12-14th type III repeats, were produced by standard recombinant DNA methodologies as previously described. ${ }^{19}$ Briefly, each DNA fragment was inserted into a pGEX-6-P1 plasmid (GE Healthcare, UK) and separately amplified in DH5 $\alpha$ cells (Invitrogen, USA). After purifying and sequencing, correct constructs were separately inserted in BL21 cells (New England BioLabs, UK) and the resulting colonies were 
dynamically cultured in LB broth containing $100 \mu \mathrm{g} / \mathrm{ml}$ ampicillin at $37^{\circ} \mathrm{C}$. Protein production was induced by isopropyl $\beta$-D-1-thiogalactopyranoside (IPTG; $1 \mathrm{mM}$ final concentration) during $4 \mathrm{~h}$ at $37^{\circ} \mathrm{C}$. Cells were harvested by centrifugation, resuspended and sonicated. After removing cell fragments and insoluble proteins by centrifugation proteins were purified using a GSTrap affinity column (GE Healthcare). GST-tag was removed on-column and purified fragments were resolved by electrophoresis. Protein concentrations were quantified by BCA method following the manufacturer's instructions (Thermo Fisher Scientific, USA).

\subsection{RGD-point Mutation}

Two missense mutations were generated in the HBII construct to introduce an RGD motif (RGD-mutated HBII fragment; Figure 1) by using the Quickchange Lightning site-directed mutagenesis kit (Agilent Technologies, USA). Mutagenesis reactions were performed in a T100 thermal cycler (Bio-Rad, USA) according to the manufacturer's guidelines. DNA primers for the P233R mutation were 5'-CT CGG CCC CGC CGT GGT GTC ACA GA-3' (forward) and 5'-TC TGT GAC ACC ACG GCG GGG CCG AG-3' (reverse). Plasmids containing the mutation were transformed into DH5 $\alpha$ cells, purified and sequenced. Correct plasmids were mutated in a second round using 5'-CC CGC CGT GGT GALC ACA GAG GCT AC-3' (forward) and 5'-GT AGC CTC TGT GTC ACC ACG GCG GG-3' (reverse) primers for the V235D mutation. Constructs were transformed into DH5 $\alpha$ cells, purified and sequenced. Details on gene sequencing and the resulting mutated sequence are shown in Supporting Information. Correct constructs were transformed into BL21 cells and RGD-mutated HBII fragments were produced and purified as explained above. 


\subsection{Covalent Attachment}

The molecules were covalently attached to Ti discs by silanization as carefully described in previous studies. ${ }^{19,24}$ Briefly, Ti discs were cleaned and activated by oxygen plasma for 5 min at $12 \mathrm{MHz}$ in a Femto low pressure plasma system (Diener electronic, Germany). Then, samples were immersed in $0.08 \mathrm{M}$ solution of (3aminopropyl)triethoxysilane (APTES, Sigma-Aldrich) at $70^{\circ} \mathrm{C}$ for $1 \mathrm{~h}$, rinsed with different solvents and cross-linked with $7.5 \mathrm{mM}$ solution of N-succinimidyl-3maleimidepropionate (SMP). Finally, fibronectin recombinant fragments were immobilized on Ti surface at a $100 \mu \mathrm{g} / \mathrm{ml}$ concentration (optimized in previous studies by our group ${ }^{19}$ ) in phosphate-buffered saline (PBS) to ensure saturation of the surface. Uncoated polished Ti discs and fibronectin-coated discs were used as negative and positive controls, respectively. Discs functionalized with $70 \%$ CAS and 30\% HBII (CAS70:HBII30) were also used as positive controls considering the results obtained in a previous study. ${ }^{19}$

\subsection{Functionalization Characterization}

\subsubsection{Surface Chemical Composition}

The chemical composition (atomic percentage) at the surface level was measured by Xray photoelectron spectroscopy (XPS; SPECS Surface Nano Analysis GmbH, Germany) to determine the presence of RGD-mutated HBII fragment after immobilization. Spectra of samples were acquired with a non-monochromatic Mg anode XR50 source operating at $150 \mathrm{~W}$ and a Phoibos $150 \mathrm{MCD}-9$ detector. Detector pass energy was fixed at $25 \mathrm{eV}$ with $0.1 \mathrm{eV}$ steps to record high resolution spectra at a pressure below $7.5 \times 10^{-9}$ mbar. Peak fittings and spectral analysis were performed using CasaXPS software (version 2.3.16, Casa Software Ltd., UK). All binding energies were calibrated with the C 1s signal located at $284.8 \mathrm{eV}$. 


\subsubsection{Biomolecule Density at the Surface}

The adsorption kinetics of the RGD-mutated HBII fragment on Ti surface was evaluated by quartz crystal microbalance with monitoring dissipation (QCM-D). For this assay, $\mathrm{TiO}_{2}$ sensors (QSX310, Q-Sense, Sweden) were silanized and coated as described above for $\mathrm{Ti}$ discs. Measurements were performed at $37^{\circ} \mathrm{C}$ by monitoring changes in frequency, $\Delta \mathrm{f}(\mathrm{Hz})$, and dissipation, $\Delta \mathrm{D}\left(\times 10^{-6}\right)$, in real-time using Qsoft software (QSense) in a D300 equipment (Q-Sense). First, PBS was used to completely stabilize the baseline during 30-60 min and then the biomolecule was injected at $100 \mu \mathrm{g} / \mathrm{ml}$ in PBS monitoring its adsorption during $280 \mathrm{~min}$. Finally, weakly bound biomolecules were rinsed with PBS for 30 min. All raw data was analyzed using QTools software (QSense) to obtain surface mass density and thickness values.

\subsection{Determination of Integrin and Growth Factor Binding Capacity}

The capacity of the biomolecules to bind growth factors was evaluated by QCM-D. To this end, Ti sensors were coated with CAS, HBII or RGD-mutated HBII as described in the previous section and blocked injecting $100 \mu \mathrm{g}$ of bovine serum albumin (BSA) in PBS to prevent non-specific protein interactions. After the baseline was stabilized, nonattached BSA was removed by rinsing in PBS for 10 min and $1 \mu \mathrm{g}$ of BMP-2 or TGF- $\beta 1$ (Peprotech, USA) were injected. Finally, non-attached growth factors were removed by rinsing with PBS for 10 min. Increases in surface mass density were quantified using QTools software (Q-Sense).

To determine the capacity of the different molecules to bind integrin $\alpha 5 \beta 1$ and BMP-2, an ELISA experiment was performed on Ti functionalized samples. To this end, Ti discs were functionalized as above described with $100 \mu \mathrm{g} / \mathrm{ml}$ of CAS, HBII or HBIIRGD fragments. The discs were rinsed thrice in $0.01 \%$ Tween-20 in PBS (PBS-T) and incubated with blocking buffer $(20 \mathrm{mM}$ Tris- $\mathrm{HCl} \mathrm{pH}$ 7.5, $150 \mathrm{mM} \mathrm{NaCl}, 1 \mathrm{mM} \mathrm{CaCl}$, 
$1 \mathrm{mM} \mathrm{MgCl} 2,1 \mathrm{mM} \mathrm{MnCl} 2$ and 1\% BSA) for $1 \mathrm{~h}$. Samples were washed thrice in PBS$\mathrm{T}$ and incubated with different concentrations of integrin $\alpha 5 \beta 1$ (ranging from 0 to 100 $\mathrm{nM}$; R\&D Systems, USA) or BMP-2 (ranging from 0 to $400 \mathrm{nM}$; Peprotech) for $2 \mathrm{~h}$. Afterwards, samples were washed four times in PBS-T and incubated with rabbit antiintegrin $\beta 1$ (1:300; Thermo Fisher Scientific) or goat anti-BMP-2 (1:100; Santa Cruz Biotechnologies) for 1h. After five washes in PBS-T, samples were incubated with goat anti-rabbit immunoglobulins/HRP or rabbit anti-goat immunoglobulins/HRP (1:2000; Agilent Dako, USA) for $1 \mathrm{~h}$. Samples were then washed five times in PBS-T and detection was performed by incubating in $100 \mu$ of 3,3',5,5'-tetramethylbenzidine (TMB; Sigma-Aldrich) substrate solution for $10 \mathrm{~min}$ before stopping the enzymatic reaction by adding $100 \mu \mathrm{L} 1 \mathrm{NH} 2 \mathrm{SO} 4$. Colorimetric intensity was then measured at 450 $\mathrm{nm}$ in a Synergy HTX multi-mode reader (Bio-Tek).

The capacity of the novel RGD-mutated HBII molecule to bind at the same time integrin $\alpha 5 \beta 1$ and BMP-2 was evaluated following a variation of the ELISA protocol. Samples functionalized with CAS, HBII or HBII-RGD were blocked, incubated with $50 \mathrm{nM}$ integrin $\alpha 5 \beta 1$ (saturating concentration determined in the previous ELISA) for $2 \mathrm{~h}$ and then incubated with $100 \mathrm{nM}$ BMP-2 solution for $2 \mathrm{~h}$. Samples were incubated with goat anti-BMP-2 and rabbit anti-goat immunoglobulins/HRP before adding TMB. The binding of BMP-2 after incubating the samples with integrin $\alpha 5 \beta 1$ was measured at 450 $\mathrm{nm}$ as above described.

\subsection{Mesenchymal Stem Cell Response}

\subsubsection{Cell Culture}

Human bone marrow mesenchymal stem cells (hMSCs; Tebu-bio, France) were cultured in Advanced DMEM supplemented with 10\% foetal bovine serum (FBS), 20 $\mathrm{mM}$ HEPES buffer solution, penicillin/streptomycin antibiotics $(50 \mathrm{U} / \mathrm{ml}$ and $50 \mu \mathrm{g} / \mathrm{ml}$, 
respectively) and $2 \mathrm{mM}$ L-glutamine (all from Thermo Fisher Scientific) at $37^{\circ} \mathrm{C}$ in a humidified atmosphere and $5 \% \mathrm{CO}_{2}$. Cells from passage 4 were used in all the experiments.

\subsubsection{Cell Adhesion and Proliferation}

Cells were seeded in serum-free conditions at a concentration of 10000 cells/sample and allowed to adhere for $4 \mathrm{~h}$. Then, medium was aspirated and cells were cultured for 28 days in complete medium containing FBS changing the medium thrice a week. After each incubation period ( 4 h, 3 days, 7 days, 14 days, 21 days and 28 days), cells were rinsed thrice in PBS and lysed with $300 \mu$ of Mammalian Protein Extraction Reagent (M-PER, Thermo Fisher Scientific). The number of living cells adhered at samples was measured quantifying the released lactate dehydrogenase (LDH) activity after lysis using the Cytotoxicity Detection Kit ${ }^{\mathrm{PLUS}}$ (LDH) (Roche, USA). Obtained absorbances at $492 \mathrm{~nm}$ in a Synergy HTX multi-mode reader (Bio-Tek, USA) were expressed as cell number using a calibration curve with increasing numbers of cells.

\subsubsection{Cell Spreading and Adhesion Interaction}

Cells (25000 cells/sample) were seeded in serum-free medium in each sample and after $4 \mathrm{~h}$ fixed with $4 \%$ paraformaldehyde (PFA) for $20 \mathrm{~min}$. Then cells were permeabilized with $0.05 \%$ Triton X-100 in PBS for 15 min and washed thrice with $20 \mathrm{mM}$ glycine in PBS (washing buffer). Samples were blocked with 1\% BSA for 30 min to prevent nonspecific protein interaction. For the cell spreading assay, cells were incubated with mouse antivinculin antibody (1:100) for $1 \mathrm{~h}$, rinsed with washing buffer and incubated with Alexa Fluor 488 goat antimouse antibody (1:1000) and Alexa Fluor 546 phalloidin (1:300) in the dark for $1 \mathrm{~h}$. For the adhesion interaction assays, cells were incubated with mouse anti-syndecan-4 (1:100; Santa Cruz Biotechnologies) and rabbit antiintegrin $\beta 1(1: 300)$ antibodies for $1 \mathrm{~h}$, rinsed with washing buffer and incubated with 
Alexa Fluor 488 chicken anti-rabbit and Alexa Fluor 568 goat anti-mouse $(1: 1000)$ antibodies in the dark for $1 \mathrm{~h}$. After antibodies incubation, nuclei were counterstained with DAPI (1:1000) and samples mounted in Mowiol 4-88 (Sigma-Aldrich, USA) before visualizing in an LSM 800 confocal laser scanning microscope (Carl Zeiss, Germany). For the spreading assay, at least five images from different areas at $10 \times$ magnification of each sample were acquired and the area of cells was calculated using the ImageJ software (National Institute of Health, USA). For the adhesion interaction assay, image stitching of $3 \times 3$ images at $63 \times$ magnification was performed.

The amount of integrin $\beta 1$ and syndecan- 4 expressed by cells was quantified by western blot analysis. To this end, cells were seeded in triplicate (30000 cells/sample) as explained above and allowed to adhere for $4 \mathrm{~h}$. Then, cells were lysed in RIPA buffer ( 50 $\mathrm{mM}$ Tris- $\mathrm{HCl} \mathrm{pH}$ 7.6, $150 \mathrm{mM} \mathrm{NaCl}, 0.25 \%$ sodium deoxycholate, $0.1 \% \mathrm{SDS}, 1 \mathrm{mM}$ EDTA, plus protease inhibitors; Sigma-Aldrich) and centrifuged at $12.000 \mathrm{~g}$ for $10 \mathrm{~min}$. Equal amounts of proteins were size-fractioned in SDS-PAGE under reducing conditions $(100 \mathrm{~V}, 2 \mathrm{~h})$ and transferred to nitrocellulose membranes (60V, overnight). Membranes were blocked with $10 \%$ BSA in PBS-T and incubated with mouse anti- $\beta$ actin (1:500; Santa Cruz Biotechnologies), rabbit anti-integrin $\beta 1$ (1:300; Thermo Fisher Scientific) or mouse anti-syndecan-4 (1:100; Santa Cruz Biotechnologies) overnight at $4^{\circ} \mathrm{C}$. After several washes in PBS-T, membranes were incubated with goat anti-rabbit immunoglobulins/HRP or goat anti-mouse immunoglobulins/HRP (Agilent Dako) and detection was performed with luminol reagent (Santa Cruz Biotechnologies). Bands were quantified with the Quantity One Quantitation Software (Bio-Rad, USA) on unsaturated x-ray films. Results were normalized versus the signal obtained for $\beta$-actin. 


\subsubsection{Cell Differentiation}

The alkaline phosphatase (ALP) activity was quantified using the same extracts obtained from the proliferation assay by a SensoLyte pNPP Alkaline Phosphatase Activity Kit (AnaSpech Inc., USA). ALP levels were obtained after extrapolating the absorbances obtained at $405 \mathrm{~nm}$ using a Synergy HTX multi-mode reader (Bio-Tek) to a calibration curve prepared following the manufacturer's instructions. Results were normalized versus their corresponding cell numbers obtained in the cell proliferation assay.

The expression of genes related to osteogenic differentiation was evaluated by real time quantitative polymerase chain reaction (RT-qPCR). Cells were cultured at a density of 25000 cells/sample on Ti discs functionalized with HBII or RGD-mutated HBII fragments and allowed to adhere for $4 \mathrm{~h}$ in serum-free medium. Non-functionalized Ti was used as control substrate. Then, cells were incubated for additional $2 \mathrm{~h}$ in serumfree medium with or without $25 \mathrm{ng} / \mathrm{ml}$ of BMP-2. After removing the medium, cells were incubated in serum-free medium until $24 \mathrm{~h}$ after cell seeding. Then, FBS was added and cells cultured for 7 days changing the medium every two days. After each incubation period (1 day, 3 days and 7 days), total RNA was extracted and purified using the RNeasy Mini Kit (Qiagen, Germany). Equal amounts of RNA (100 ng), quantified using a NanoDrop ND-1000 spectrophotometer (NanoDrop Technologies, USA), were retrotranscribed to cDNA using the QuantiTect Reverse Transcription Kit (Qiagen). RT-qPCR analyses were performed using the QuantiTect SYBR Green RTPCR Kit (Qiagen) and specific primers (Table 1) in a StepOnePlus Real-Time PCR System (Applied Biosystems, USA). The fold change of gene expression was obtained after normalizing the values to Ti without BMP-2 at day 1 and to $\beta$-actin. Detailed protocol and calculations has been described elsewhere. ${ }^{25}$ 
Table 1. DNA primer sequences for the genes related to osteogenic differentiation used for RT-qPCR.

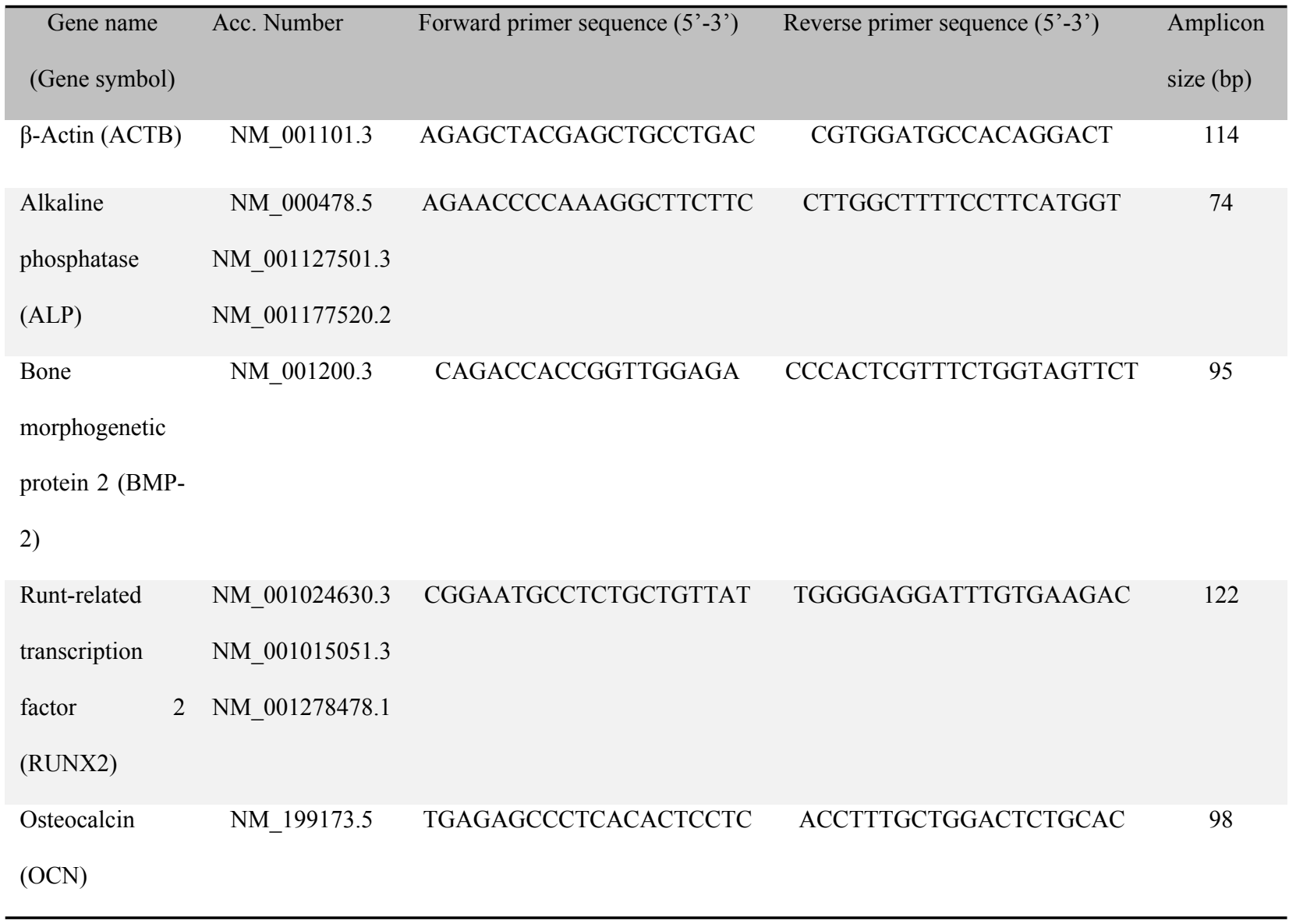

\subsubsection{Cell Mineralization}

Cells were seeded in the same conditions described for the cell proliferation assays for 14, 21 and 28 days. Then, cells were fixed with 4\% PFA for $15 \mathrm{~min}$ and washed twice with Milli-Q water. Calcium deposits were stained with $500 \mu 1 /$ sample of $40 \mathrm{mM}$ Alizarin Red S (Sigma-Aldrich) for 20 min with gently shaking. After removing excess dye with several Milli-Q water washings, stained surfaces at 28 days of culture were examined using an Olympus BX51-P microscope (Olympus Corp., Japan). Afterwards, $10 \%$ cetylpyridinium chloride in $10 \mathrm{mM} \mathrm{NaH} 2 \mathrm{PO} 4$ was added to extract the staining. Supernatants were then collected and spectrophotometrically measured at $570 \mathrm{~nm}$ in a 
Synergy HTX multi-mode reader (Bio-Tek). Results were normalized versus their corresponding cell numbers obtained in the cell proliferation assay.

\subsection{Statistical Analysis}

All data are presented as mean values \pm standard error of the mean. Experiments were performed in triplicate using in each one three replicates per each group. Statistically significant differences between groups ( $p$-value $<0.05$ ) were analyzed by KruskalWallis non-parametric test followed by Mann-Whitney test with Bonferroni correction using the SPSS statistics software (IBM, USA).

\section{RESULTS}

An RGD sequence was included in the HBII protein sequence by modifying the DNA sequence with two missense mutations (see Materials and Methods). The mutations were produced in a loop in the $\mathrm{FN} \mathrm{III}_{14}$ domain (Figure $1 \mathrm{~A}$ ) which is closely similar to the RGD loop in the CAS domain in order to be sensed by cells. Herein, a PGV amino acid sequence was mutated to RGD by modifying only two nucleotides (Figure 1B). 
A
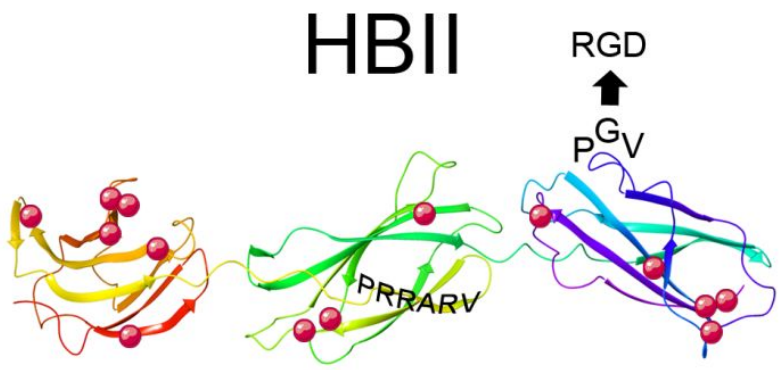

$\mathrm{FN} \mathrm{III}_{12}$

$\mathrm{FN} \mathrm{III}_{13}$

$\mathrm{FN} \mathrm{III}$

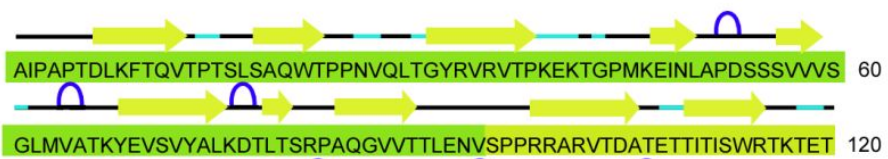
GLMVATKYEVSVYALKDTLTSRPAQGVVTTLENVSPPRRARVTDATETTITISWRTKTET 120

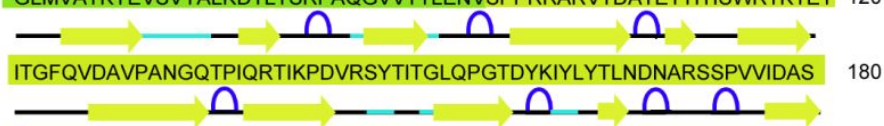
TAIDAPSNLRFLATTPNSLLVSWQPPRARITGYIIKYEKPGSPPREWPRPRPGVTEATI 240 $\frac{0}{\text { TGLEPGTEYTIYVIALKNNQKSEPLIGRKKT }} 271$
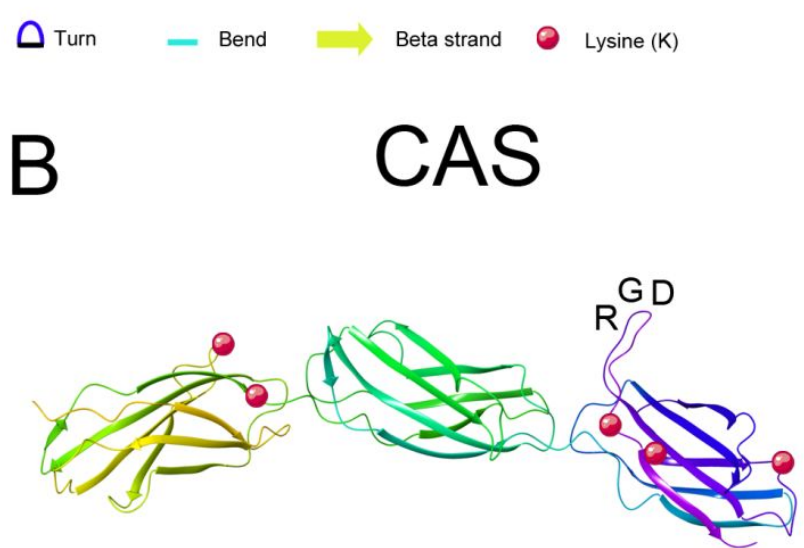

$\mathrm{FN} \mathrm{III}_{8} \quad \mathrm{FN} \mathrm{III} \mathrm{F}_{9} \quad \mathrm{FN} \mathrm{III}_{10}$

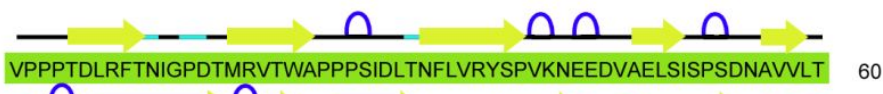
VPPPTDLRFTNIGPDTMRVTWAPPPSIDLTNFLVRYSPVKNEEDVAELSISPSDNAVVLT 60 $\frac{\Omega}{\text { NLLPGTEYVVSVSSVYEQHESTPLRGRQKTGLDSPTGIDFSDITANSFTVHWIAPRATIT }}$

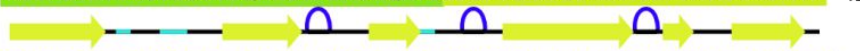
GYRIRHHPEHFSGRPREDRVPHSRNSITLTNLTPGTEYVVSIVALNGREESPLLIGQQST 180 $\underset{\text { VSDVPRDLEWVATTPTSLLISWDAPAVTVRYYRITYGETGGNSPVQEFTVPGSKSTATIS }}{0}$ $\frac{\mathrm{O}}{\text { GLKPGVDYIITYYAVTGRGDSPASKPISINYRT }} 274$

$\Delta$ Turn $\quad-$ Bend $\longrightarrow$ Beta strand $\odot$ Lysine $(K)$

Figure 1. RGD mutation in the HBII domain of fibronectin. (A) Stereoview of the folding topology of the native HBII fragment of fibronectin (top). The structure is represented as a cartoon with the $\beta$-strands shown as arrows. The sequence PRRARV in 
FN $\mathrm{III}_{13}$, responsible of the heparin binding activity has been highlighted. The Pro233Gly234-Val235 (PGV) sequence in $\mathrm{FN} \mathrm{III}_{14}$ has been mutated into Arg233-Gly234Asp235 (RGD). Lysine residues, which participate in the covalent attachment to Ti, are highlighted with red circles. Linear amino acid sequence of the HBII fragment of fibronectin (bottom). The $\beta$-strands are represented by arrows and the type III repeats are highlighted with different colours. The mutated amino acids to generate and RGD sequence are highlighted in red. (B) Stereoview of the folding topology of the native CAS fragment of fibronectin (top) and linear amino acid sequence (bottom). Lysine residues, which participate in the covalent attachment to $\mathrm{Ti}$, are highlighted with red circles. The RGD sequence is highlighted in red.

\subsection{Characterization of the Titanium Functionalization}

The presence of RGD-mutated HBII fragment at the Ti surface was analyzed measuring the surface elemental composition by XPS. A decrease of both Ti $2 p$ and $\mathrm{O} 1 \mathrm{~s}$ was observed after silanization which was also observed after functionalization (Table 2). Silanization with APTES also produced an increase in the Si 2p, N 1s and C1s. Presence of $\mathrm{N}$ was attributed to the imide group of the SMP crosslinker. The addition of RGDmutated HBII fragment produced a further increase in $\mathrm{N} 1 \mathrm{~s}$ and $\mathrm{C} 1 \mathrm{~s}$ and a considerable decrease in Si 2p. This increase of $\mathrm{N}$ can be attributed to the peptide bonds and amino acid side chains.

Table 2. Surface elemental composition (atomic percentages).

\begin{tabular}{lccccc}
\hline & Ti $2 \mathrm{p}$ & $\mathrm{O} \mathrm{1s}$ & Si 2p & N 1s & C 1s \\
Ti & $13.52( \pm 0.51)$ & $48.18( \pm 5.93)$ & $0.61( \pm 0.16)$ & $1.06( \pm 0.17)$ & $35.65( \pm 6.44)$ \\
Silanized Ti & $0.49( \pm 0.50)$ & $26.27( \pm 1.73)$ & $12.94( \pm 0.52)$ & $8.31( \pm 0.09)$ & $51.70( \pm 1.80)$ \\
Sil. Ti + RGD-mut. HBII & $0.79( \pm 0.06)$ & $20.43( \pm 0.17)$ & $3.89( \pm 0.13)$ & $14.41( \pm 0.29)$ & $60.47( \pm 0.05)$ \\
& & & & &
\end{tabular}


In addition, the presence of RGD-mutated HBII recombinant fragment on the Ti surface was quantified using QCM-D. After rinsing with PBS, the thickness and surface mass density values of the adlayer of immobilized molecule on silanized Ti sensors were close to CAS and HBII values obtained in a previous study (Table 3). ${ }^{19}$ Changes in frequency and dissipation recorded during the adsorption of RGD-mutated HBII fragment are shown in Figure $2 \mathrm{~A}$ and $2 \mathrm{~B}$, respectively. It can be noted that the frequency rapidly decreased after injection and, after rinsing with PBS, the values were stabilized.

Table 3. Thickness and surface mass density of the bound biomolecules to silanized Ti sensors characterized by quartz crystal microbalance with dissipation (QCM-D) monitoring. *CAS and HBII values were obtained in a previous study. ${ }^{*}$

\begin{tabular}{lcc}
\hline Biomolecule & Thickness & Surface mass density $(\mathrm{ng} / \mathrm{cm} 2)$ \\
CAS & $5.54( \pm 0.09)^{*}$ & $609.14( \pm 10.19)^{*}$ \\
HBII & $7.49( \pm 0.13)^{*}$ & $823.80( \pm 14.00)^{*}$ \\
RGD-mutated HBII & $5.08( \pm 0.25)$ & $559.90( \pm 27.5)$ \\
\hline
\end{tabular}



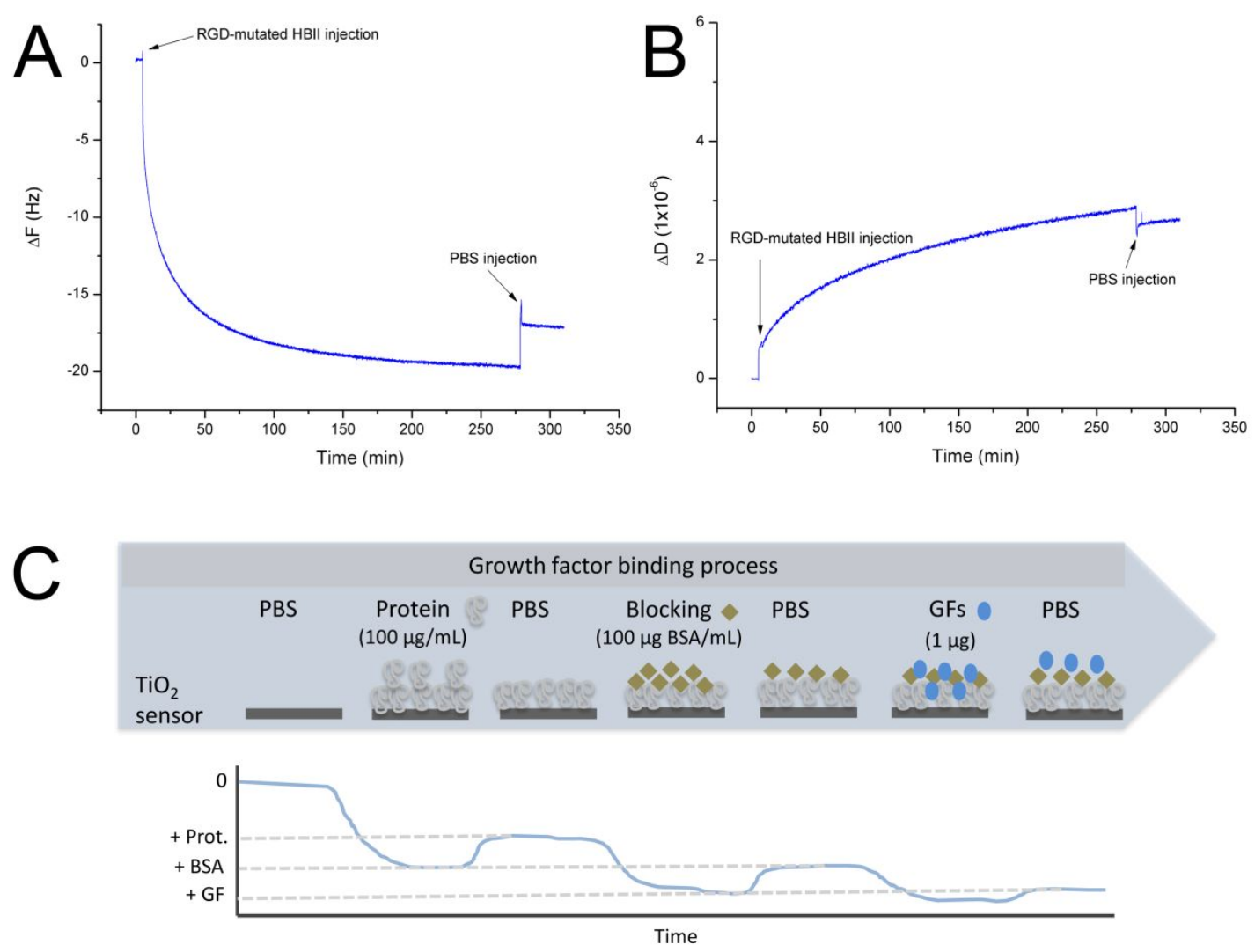

Figure 2. Adsorption kinetics of the RGD-mutated $\mathrm{HBII}$ fragment on $\mathrm{TiO}_{2}$ sensors. Frequency shift (A) and dissipation shift (B) versus time plots of RGD-mutated HBII fragment immobilized on silanized $\mathrm{TiO}_{2}$ sensors using QCM-D. (C) Representative illustration of the growth factor binding assay performed using a QCM-D. $\mathrm{TiO}_{2}$ sensors were coated with CAS, HBII or RGD-mutated HBII fragments, rinsed in PBS and blocked with BSA. After rinsing, BMP-2 or TGF- $\beta 1$ were injected and changes in the frequency of vibration were registered as increases in surface mass density.

\subsection{Integrin and Growth Factor Binding Capacity}

The interaction of the growth factors with the fibronectin recombinant fragments immobilized on Ti sensors was quantified using the QCM-D. A detailed illustration of the process is represented in Figure $2 \mathrm{C}$. An increase in the surface mass density was observed when the Ti sensors functionalized with HBII fragment were exposed to TGF- 
$\beta 1$ or BMP-2 containing solutions (Table 4). This was not observed for the CASfunctionalized Ti sensors, which presented a slight decrease in the surface mass density. Interestingly, a considerable increase in the surface mass density was observed in the surfaces functionalized with RGD-mutated HBII fragment compared to native HBII fragment exposed to TGF- $\beta 1$ containing solutions. In contrast, when RGD-mutated coated sensors were exposed to BMP-2 solutions the values were lower compared to HBII fragment, although they were positive.

Table 4. Increases in the surface mass density after depositing TGF- $\beta 1$ or BMP-2 onto functionalized Ti sensors.

\begin{tabular}{cc}
\hline Molecules & $\Delta$ Surface mass density $(\mathrm{ng} / \mathrm{cm} 2)$ \\
HBII + TGF- $\beta 1$ & $7.04( \pm 0.03)$ \\
CAS + TGF- $\beta 1$ & $-40.26( \pm 0.13)$ \\
HBII-RGD + TGF- $\beta 1$ & $116.49( \pm 2.45)$ \\
HBII + BMP-2 & $30.36( \pm 0.16)$ \\
CAS + BMP-2 & $-2.31( \pm 0.06)$ \\
HBII-RGD + BMP-2 & $14.19( \pm 0.09)$ \\
\hline
\end{tabular}

The capacity to bind integrin $\alpha 5 \beta 1$ and BMP-2 was also evaluated by ELISA. No binding of integrin $\alpha 5 \beta 1$ to native HBII was detected, since the absorbance values in all the integrin $\alpha 5 \beta 1$ concentrations were always close to the background (data not shown). Addition of RGD into the HBII sequence provided this fragment with the ability to bind integrin $\alpha 5 \beta 1$, although at lower levels compared to the CAS fragment (Figure 3A). Regarding the BMP-2 binding capacity, the HBII-RGD fragment showed lower levels compared to the native HBII fragment (Figure 3B). However, the absorbance levels showed a high level of binding to HBII-RGD fragment compared to CAS fragment, in 
which absorbance values were close to the background for all the tested BMP-2 concentrations (data not shown).
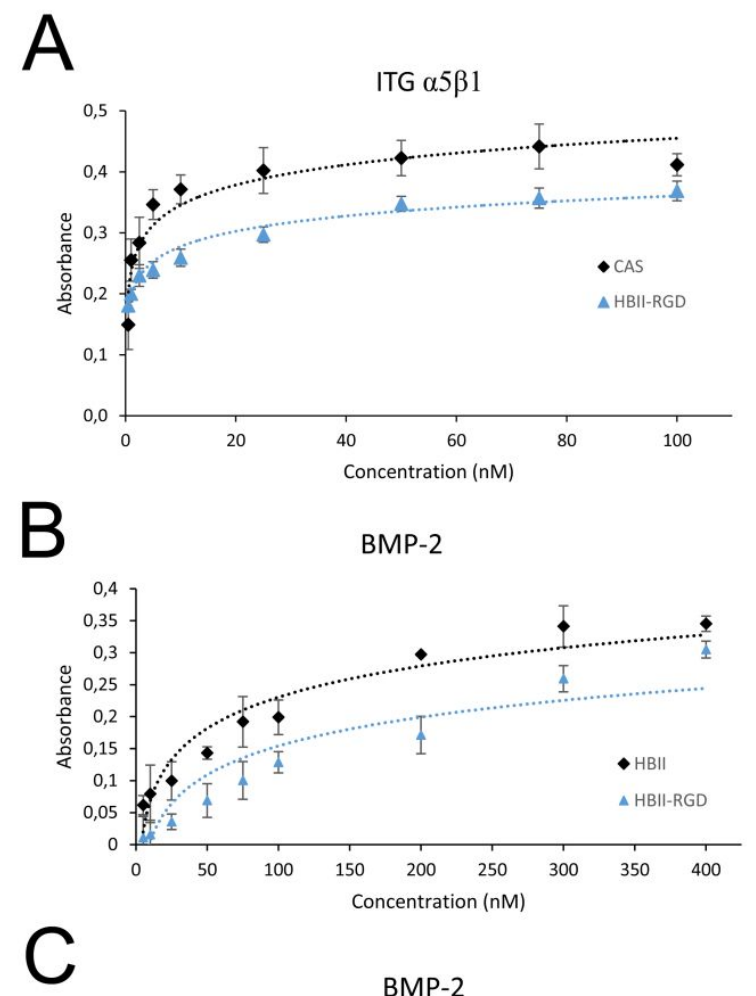

BMP-2

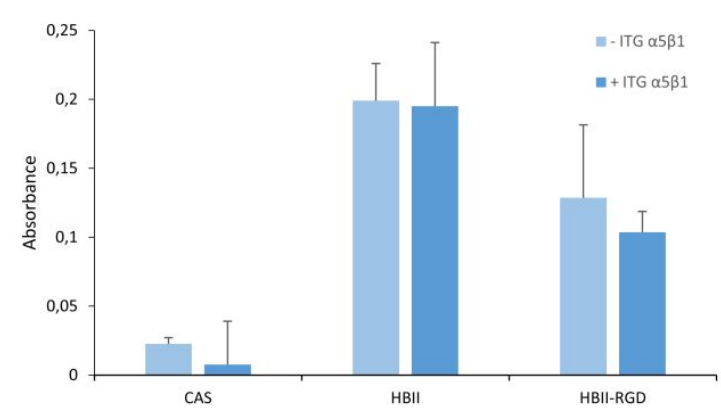

Figure 3. Determination of the interaction of fibronectin fragments with integrin $\alpha 5 \beta 1$ and BMP-2. Dose response curves for integrin $\alpha 5 \beta 1$ (A) and BMP-2 (B) and absorbance levels (C) for BMP-2 incubated on functionalized samples after binding with $(+$ ITG $\alpha 5 \beta 1$ ) or without (- ITG $\alpha 5 \beta 1$ ) integrin $\alpha 5 \beta 1$.

The capacity of the HBII-RGD molecule to bind at the same time integrin $\alpha 5 \beta 1$ and BMP-2 molecules was determined by ELISA. To this end, the functionalized surfaces were incubated with saturating integrin $\alpha 5 \beta 1$ concentrations determined from Figure $3 \mathrm{~A}$ 
$(50 \mathrm{nM})$ and afterwards incubated with a BMP-2 concentration that gave high levels in Figure 3B $(100 \mathrm{nM})$. No binding of BMP-2 was observed in CAS-functionalized surfaces (Figure 3C). In contrast, no significant differences on the levels of BMP-2 binding were observed for HBII and HBII-RGD functionalized surfaces with or without the previous incubation with integrin $\alpha 5 \beta 1$. Nonetheless, as above mentioned, the levels were lower for the HBII-RGD compared to native HBII.

\subsection{Mesenchymal Stem Cell Response}

Cells were completely spread on $\mathrm{Ti}$ surfaces functionalized with either full-length fibronectin or with the CAS fragment, presenting well-developed actin stress fibers and focal adhesion formation (Figure 4A and 4B). Nonetheless, whereas such focal adhesions were homogenously distributed in the fibronectin-coated surfaces, in those functionalized with the CAS fragment vinculin spots were mostly observed at the edges of cells. In contrast, in the HBII functionalized surfaces the cells were round with poorly organized actin filaments and no sign of focal adhesions, presenting also high amount of filopodia extensions. Interestingly, the behaviour of the cells on CAS70:HBII30 functionalized $\mathrm{Ti}$ surfaces was a combination of the patterns observed in full-length fibronectin, CAS and HBII fragments. The presence of RGD in the HBII domain did not allow complete spreading of cells, but seemed to stimulate the formation of focal adhesions at the edges of cells, where filopodia were spikier compared to HBII. In addition, formation of immature actin filaments was observed in RGD-mutated HBII condition. The cells were completely round, with no detection of actin filaments and vinculin staining when cultured on bare Ti samples. 


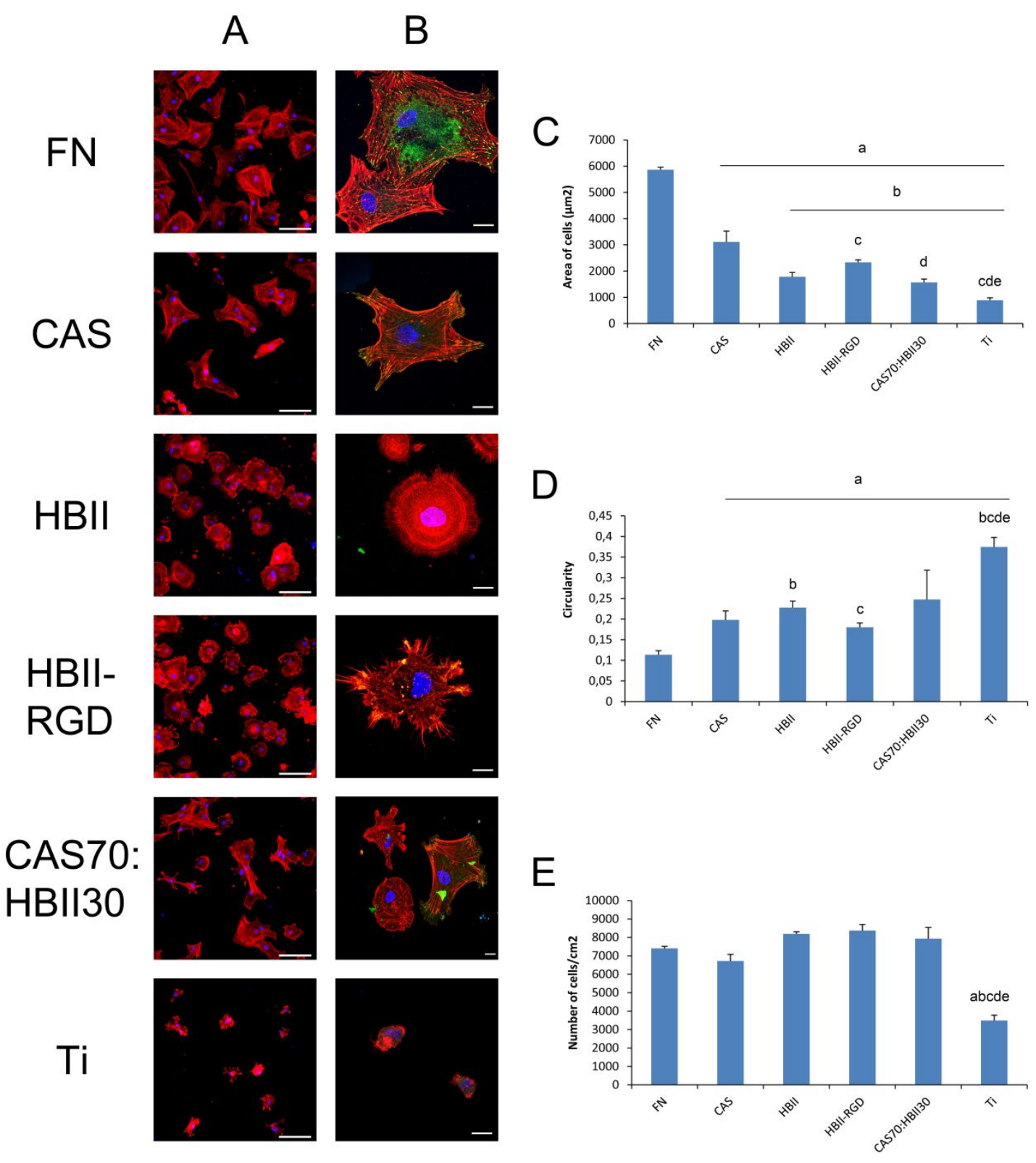

Figure 4. Cell adhesion on the different functionalized surfaces. Representative (A) low magnification (10x) and (B) high magnification (63x) images of hMSCs spreading and morphology after $4 \mathrm{~h}$ of adhesion on functionalized Ti with fibronectin (FN), CAS, HBII, CAS70\%:HBII30\% (CAS70:HBII30), RGD-mutated HBII (HBII-RGD) and nonfunctionalized $\mathrm{Ti}$ (Ti). Scale bare denotes $50 \mu \mathrm{m}$ and $10 \mu \mathrm{m}$, respectively. Area (C), cell number (D) and circularity (E) of hMSCs adhered after $4 \mathrm{~h}$ of culture on the different functionalized $\mathrm{Ti}$ surfaces. Letter $a$ indicates statistically significant differences 
compared to $\mathrm{FN}$, letter $b$ indicates statistically significant differences compared to CAS, letter $c$ indicates statistically significant differences compared to HBII, letter $d$ indicates statistically significant differences compared to RGD-mutated HBII (HBII-RGD) and letter $e$ indicates significant differences compared to CAS70\%-HBII30\% $(p<0.05)$.

The differences observed in morphology were quantified by measuring the area of cells (Figure 4C). The highest cell spreading was observed in fibronectin surfaces whereas the lowest values were observed on bare $\mathrm{Ti}$, which were the positive and negative controls respectively. Interestingly, the presence of RGD in the HBII domain stimulated cell spreading compared to native HBII, presenting intermediate values within CAS and HBII and very close to CAS70:HBII30. In contrast, circularity values were in opposite direction. The highest value was observed for cells cultured on $\mathrm{Ti}$, whereas the lowest value was obtained on FN coated surfaces (Figure 4D). Noteworthy, RGD mutation on the HBII fragment reduced the circularity values compared to native HBII fragment, presenting comparable values to CAS-coated surfaces. Interestingly, the number of adhered cells was similar in all the functionalized surfaces, with no statistically significant differences between all groups, except bare Ti (Figure 4E).

The interaction of hMSCs with the RGD motif produced in the RGD-mutated HBII fragment was evaluated by staining the proteins involved in cell adhesion, i.e. integrin $\beta 1$ and syndecan- 4 (Figure 5A and 5B). Both integrin $\beta 1$ and syndecan- 4 were detected on the fibronectin and CAS70:HBII30 functionalized surfaces. However, whereas in presence of fibronectin all cells presented homogeneous staining throughout the membrane, in CAS70:HBII30 functionalized surfaces only some cells presented diffuse staining. In contrast, cells cultured on CAS or HBII functionalized surfaces were completely stained with either only integrin $\beta 1$ or only syndecan- 4 , respectively. Interestingly, the presence of RGD in the RGD-mutated HBII functionalized samples 
stimulated the clustering of integrin $\beta 1$ molecules, which were only noticed at the extended filopodia.

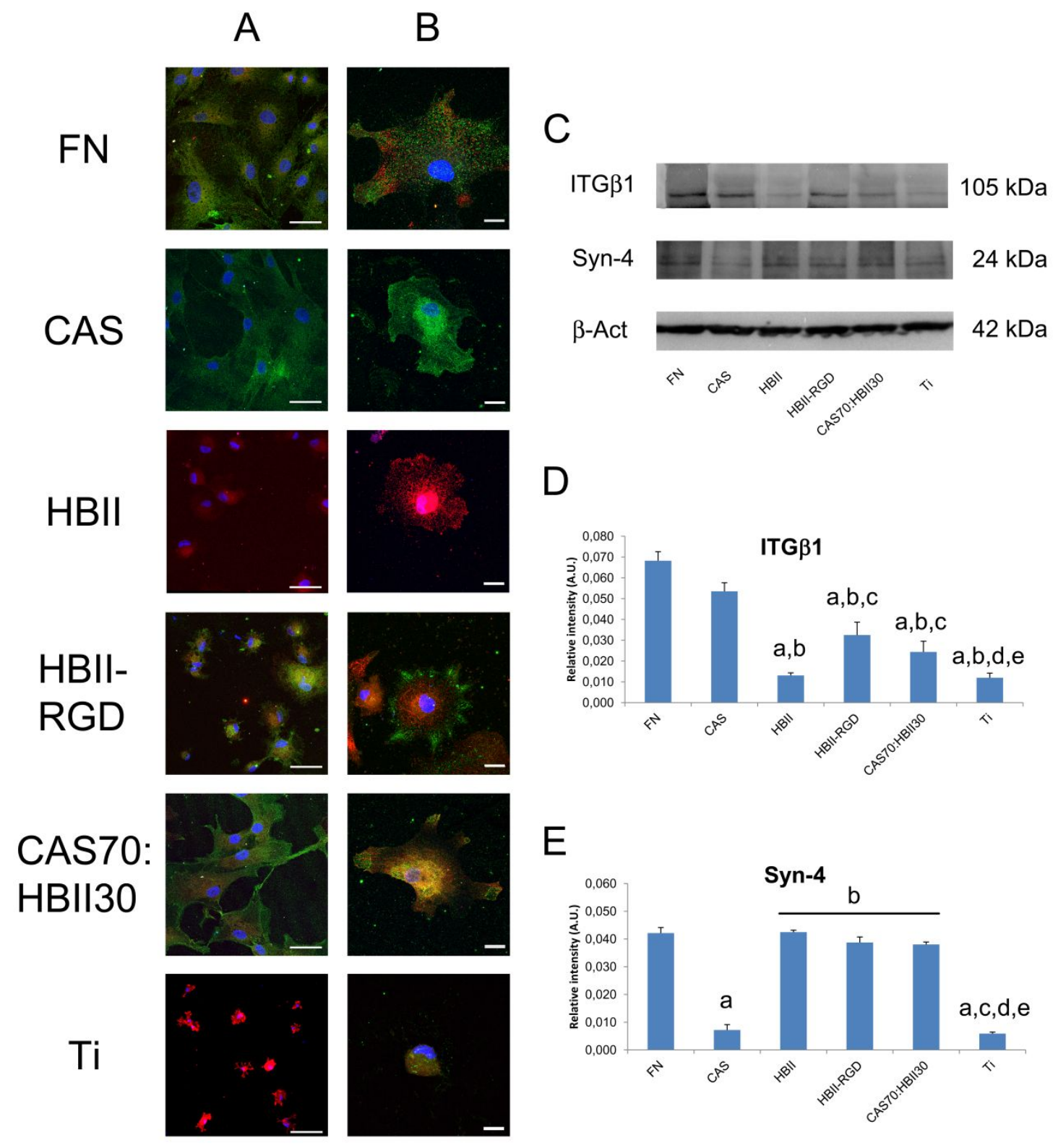

Figure 5. Expression of integrin $\beta 1$ and syndecan- 4 by cells cultured on the different functionalized surfaces. Stitching (A) and high magnification (B) immunofluorescence images of integrin $\beta 1$ (green) and syndecan-4 (red) of hMSCs cultured on fibronectin (FN), CAS, HBII, CAS70\%-HBII30\% (CAS70:HBII30), HBII-RGD and titanium (Ti) surfaces. Scale bar denotes $50 \mu \mathrm{m}$ and $10 \mu \mathrm{m}$, respectively. Representative images for 
the western blot detection (C) and quantification of integrin $\beta 1$ (D) and syndecan-4 (E). Letter a indicates statistically significant differences compared to FN, letter b indicates statistically significant differences compared to CAS, letter c indicates statistically significant differences compared to HBII, letter d indicates statistically significant differences compared to RGD-mutated HBII (HBII-RGD) and letter e indicates significant differences compared to CAS70\%-HBII30\% $(\mathrm{p}<0.05)$.

The amount of integrin $\beta 1$ and syndecan- 4 proteins expressed by cells was quantified by western blot (Figure 5C). Integrin $\beta 1$ levels were higher on cells cultured on FN and CAS compared to cells cultured on the other surfaces (Figure 5D). Interestingly, the presence of RGD on HBII stimulated the expression of integrin $\beta 1$. Syndecan- 4 was expressed by cells cultured on all the functionalized surfaces except on CAS (Figure $5 \mathrm{E})$.

The capacity of hMSCs to proliferate on the different functionalized substrates was studied after culturing cells for 3, 7, 14, 21 and 28 days (Figure 6A). The molecules containing the CAS domain (i.e. fibronectin and CAS) stimulated the highest values of proliferation at 21 days, whereas HBII domains including the RGD-mutated HBII domain produced lowest cell proliferation values, equivalent to uncoated Ti.

Opposite trends were observed for the ALP activity values (Figure 6B). The highest values were obtained when hMSCs were cultured onto HBII and RGD-mutated HBII functionalized Ti surfaces. Noteworthy, when comparing both functionalizations, the RGD-mutated HBII stimulated statistically significant higher ALP production at 28 days. In contrast, hMSCs produced lower ALP quantities when cultured on surfaces functionalized with CAS, fibronectin and non-functionalized Ti. CAS70:HBII30 presented intermediate values between HBII and CAS containing fragments. 


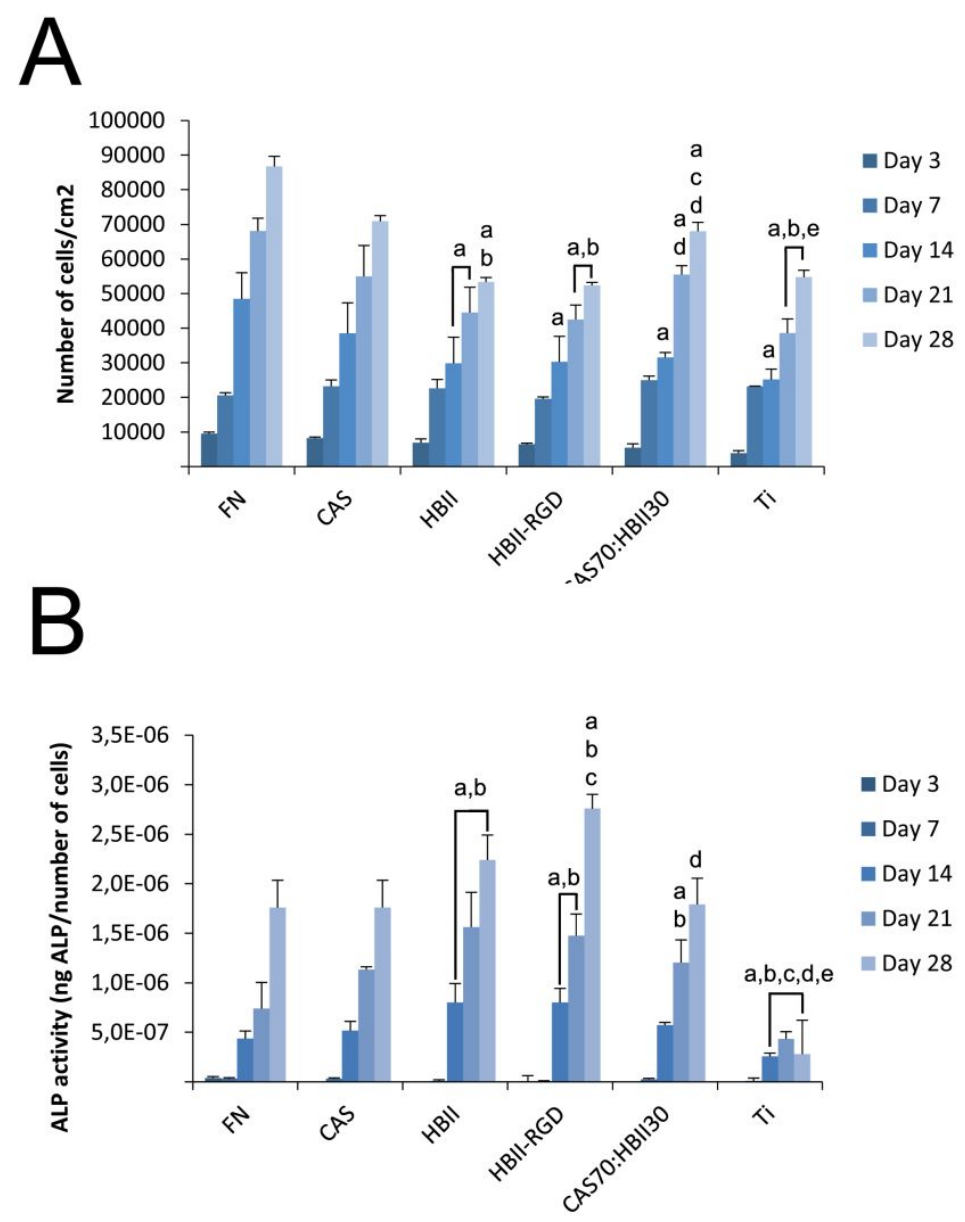

Figure 6. Cell proliferation and differentiation on the different functionalized surfaces. LDH (A) and ALP (B) activity of cells cultured on the different functionalized Ti surfaces for 3 days, 7 days, 14 days, 21 days and 28 days. At each time point, letter $a$ indicates significant differences compared to $\mathrm{FN}$, letter $b$ indicates significant differences compared to CAS, letter $c$ indicates significant differences compared to HBII, letter $d$ indicates significant differences compared to RGD-mutated HBII (HBIIRGD) and letter $e$ indicates significant differences compared to CAS70-HBII30 ( $p<$ $0.05)$.

Cell differentiation was also evaluated by measuring the expression of genes related to osteogenesis. In general, similar trends were observed for all the analyzed genes (Figure 7). Specifically, the ALP, BMP-2, RUNX2 and OCN gene expression values considerably increased when cells were cultured for 1 day on HBII functionalized 
surfaces in absence of BMP-2 compared to control substrate (Ti). Noteworthy, the observed increase for all genes at 1 day was higher when cells were cultured on RGDmutated HBII functionalized Ti surfaces in absence of BMP-2 in the medium compared to native HBII fragment. Then, gene expression levels notably decreased in both HBII and RGD-mutated HBII surfaces, excepting OCN that slowly decreased during cell culture time. Interestingly, when cells were cultured for $2 \mathrm{~h}$ with BMP-2 the gene expression levels for all the analyzed genes increased at 1 day of culture in both HBII and RGD-mutated HBII functionalized Ti surfaces compared to results obtained in absence of BMP-2. At day 3 and day 7 gene expression values also decreased to control levels, presenting OCN slower decreases. In contrast, the values for all genes in cells cultured on $\mathrm{Ti}$ were similar during all the cell culture time in absence of BMP-2, observing no significant increases. Conversely, gene expression for BMP-2, RUNX2 and OCN slightly increased on Ti when cells were cultured in presence of BMP-2.
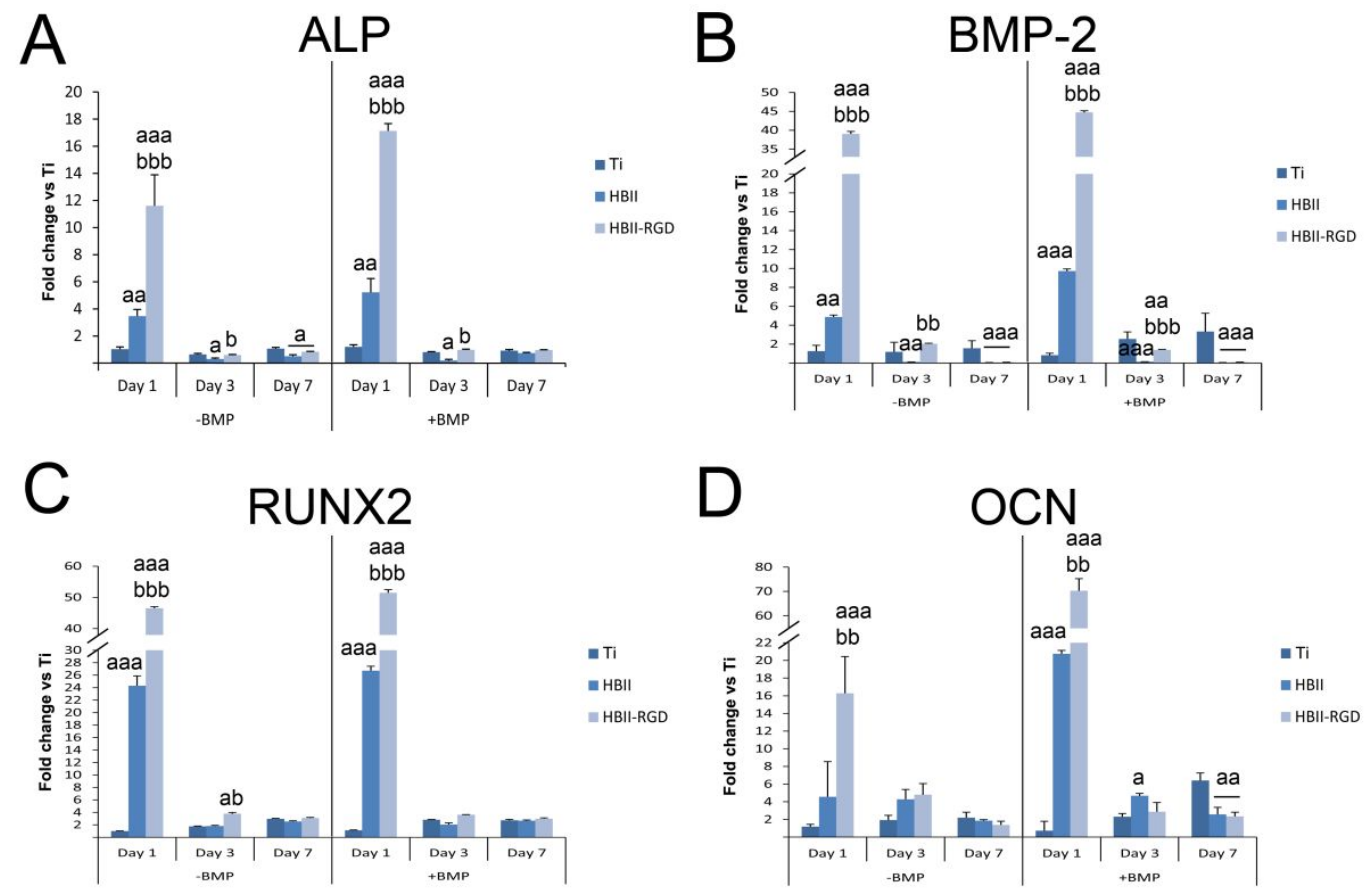

Figure 7. Gene expression levels for ALP (A), BMP-2 (B), RUNX2 (C) and OCN (D) of hMSCs cultured with or without BMP-2 on control surfaces (Ti) or HBII or RGD- 
mutated HBII functionalized surfaces for 1, 3 and 7 days. Results were normalized versus $\beta$-actin and are represented as relative fold change compared to Ti without BMP2 at day 1. At each time point, letter a indicates significant differences compared to FN, letter $\mathrm{b}$ indicates significant differences compared to CAS, letter $\mathrm{c}$ indicates significant differences compared to HBII, letter d indicates significant differences compared to RGD-mutated HBII (HBII-RGD) and letter e indicates significant differences compared to CAS70-HBII30 ( $p<0.05)$. Two letters indicate $p<0.01$ and three letters indicate $p<$ 0.001 .

The capacity of the different functionalized Ti surfaces to induce mineralization was evaluated by quantifying the deposition of calcium by Alizarin Red staining (Figure 8). The highest levels were observed for the surfaces containing the HBII fragment, i.e. RGD-mutated HBII, HBII and CAS70-HBII30. Noteworthy, the highest staining values were observed in cells cultured on RGD-mutated HBII surfaces, being almost three times higher (Figure 5D and 5E). The mineralization levels were lower in CAS and FN surfaces compared to surfaces containing the HBII fragment, bare Ti surfaces presenting the lowest values.

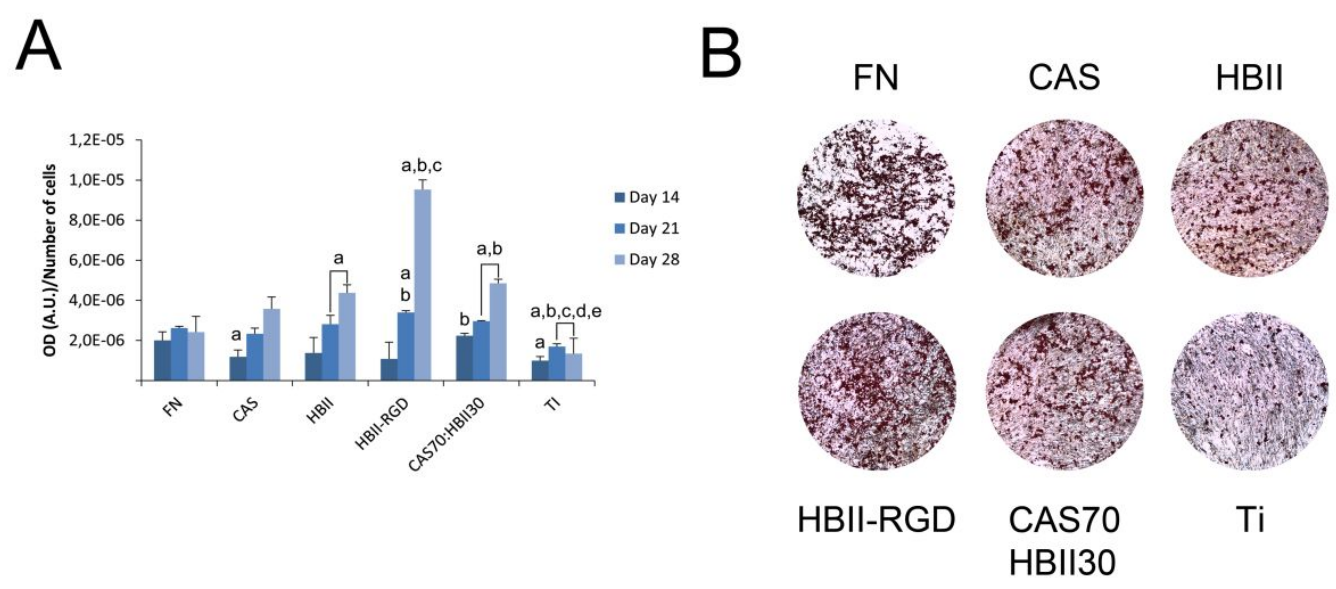

Figure 8. Mineralization of cells cultured on the different functionalized surfaces. Quantification of calcium deposits (A) produced by hMSCs on the different 
functionalized Ti surfaces after extracting Alizarin Red staining at 14, 21 and 28 days of culture. Results were normalized versus their corresponding cell numbers. Representative optical microscopy images (B) acquired at 28 days of culture. At each time point, letter a indicates significant differences compared to FN, letter b indicates significant differences compared to CAS, letter c indicates significant differences compared to HBII, letter d indicates significant differences compared to RGD-mutated HBII (HBII-RGD) and letter e indicates significant differences compared to CAS70HBII30 ( $<<0.05)$.

\section{DISCUSSION}

Mutations are naturally occurring changes in the DNA genomic sequence that can lead to alterations in protein functions. Since mutation events introduce random genetic changes, most of the time they result in loss of function. It is therefore not surprising that in most cases mutations are associated with a negative process, such as cancer. These loss-of-function mutations are traditionally used in the laboratory for impairing protein functionality or identifying functional sequences. Nonetheless, sometimes the random change by pure chance confers some new function on the gene. These gain-offunction mutations may provide selective advantages that are the engine of evolution. Herein, we proposed to produce a gain-of-function directed mutation to generate a novel protein with dual activities to promote osteogenesis: stimulate cell adhesion and cell differentiation simultaneously. For this purpose, the HBII fragment was selected for its well-known ability to sequester growth factors ${ }^{20}$ that, in the end, stimulate cell differentiation into the osteoblastic lineage. Initially, we identified several regions where an RGD sequence, which should confer cell adhesion properties to the HBII domain, could be introduced by minimally modifying the DNA sequence (i.e. only two point 
mutations). In turn, such mutation should not alter or affect important sequences in the HBII protein structure.

The HBII domain consists of three type III repeats $\left(\mathrm{III}_{12,13,14}\right)$ of 90 amino acids each. Heparin binding activity is mainly located in the type $\mathrm{III}_{13}$ repeat, ${ }^{26,27}$ specifically in the sequence PRRARV defined as heparin-binding consensus sequence X-B-B-X-B-X where $\mathrm{B}$ is a basic residue and $\mathrm{X}$ is a hydrophobic residue. ${ }^{28}$ Heparin interacts with fibronectin mainly at this sequence and is involved in the interaction with other proteins containing heparin-binding domains, including growth factors, thereby acting as reservoir that is dynamically remodelled as cellular requirements change. ${ }^{29,30}$ In the present work we did not want to affect the main activity of the HBII domain, thereby the type $\mathrm{III}_{13}$ repeat was avoided in the mutation design.

Among the other two type III repeats of the HBII domain, we identified a loop in the $\mathrm{III}_{14}$ that is closely similar to the loop on the CAS domain $\mathrm{III}_{10}$ (where RGD is present, Figure 1B top and bottom) that could be sensed by cells. Therein, we modified only two nucleotides (Figure S1) to produce a change in the protein sequence from PGV to RGD (Figure 1A top and bottom). In addition, considering that silanization mainly occurs through lysine $(\mathrm{K})$ residues and most of them are opposite to the RGD mutation (Figure 1A top), we assumed that the generated RGD sequence is exposed and available for cells to interact. In fact, this was corroborated by the capacity of the novel protein fragment to bind integrin $\alpha 5 \beta 1$. Although only two amino acids were modified in the HBII domain, which is approximately 300 amino acids long, those changes may produce structural alterations in the tertiary structure of the recombinant protein. Obviously, this modification was far enough not to affect the main heparin-binding activity located at the III13 module, but might affect other HBII properties. Apart from the heparin-binding activity attributed to PRRARV in the $\mathrm{III}_{13}$ module, it has been 
recently observed that HBII binds a huge panel of growth factors with moderately highaffinity in a heparin-independent manner. ${ }^{20}$ This property is very attractive for the design of smart materials that allow reducing growth factor doses while improving their safety. ${ }^{31,32}$ Although growth factors are potent regenerative biomolecules, there are still concerns on their medical use mainly due to undesired side effects. For instance, BMP-2 is the only Food and Drug Administration (FDA)-approved osteoinductive growth factor used in biomaterials, although it may mediate postoperative inflammation, ectopic bone formation and/or osteoclast-mediated bone resorption. ${ }^{9,33}$ Therefore, the use of biomaterials functionalized with biomolecules, e.g. HBII, that sequester and release in a controlled manner endogenous growth factors could be of paramount interest for restoring tissue functions without side effects. For the specific case of HBII, it has been demonstrated that co-presentation with CAS fragment (containing at least type $\mathrm{III}_{9-10}$ modules) is required to achieve optimal in vitro and in vivo responses. ${ }^{18,19}$ However, both fragments engineered in one molecule generate a big protein that could be more sensitive to proteolytic degradation. The rationale in the present study was to generate a small molecule containing both cell adhesion (i.e. RGD mutation) and cell differentiation (i.e. growth factor retention) capacities.

The specific sequences of the HBII fragment of fibronectin responsible for the growth factors binding capacity in a heparin-independent manner remain currently undefined. In fact, there are no evidences that the PRRARV is responsible for this growth factor binding capacity in absence of heparin. For this reason, after mutating the DNA sequence of the HBII domain, we wanted to check if the recombinant protein still retained its growth factor binding capacity. To this end, we used BMP-2 and TGF- $\beta 1$ as growth factor models due to their important roles in osteogenesis processes. Both growth factors signalling transduction converges at the Runx2 gene to control 
mesenchymal precursor cell differentiation. ${ }^{34-36}$ We observed that the novel protein still retains the capacity to bind both BMP- 2 and TGF- $\beta 1$ although the presence of RGD modified to some extent the growth factor binding capacity of native HBII. Specifically, a slightly decrease in BMP-2 and a considerable increase in TGF- $\beta 1$ binding capacity was observed. Interestingly, binding of BMP-2 was not affected by the previous bonding with integrin $\alpha 5 \beta 1$ in the HBII-RGD protein fragment as observed by ELISA, which demonstrate that the novel molecule is capable to bind at the same time integrin $\alpha 5 \beta 1$ and BMP-2, although the HBII fragment is a relatively small molecule (approximately $30 \mathrm{kDa}$ ). This is possible considering the fact that not all the molecule is necessary for protein-protein interaction, as it occurs for instance between BMP-2 and its receptors. The alteration in BMP-2 and TGF- $\beta 1$ binding capacity may be attributed to structural or polarity changes induced by the presence of RGD. It is important to mention here that the experiments in the present manuscript were performed in absence of heparin. Then, we speculate that the mutation is disrupting an important region for growth factor binding in a heparin-independent manner. Although the global protein net charge did not vary, positive (Arg) and negative (Asp) charges were introduced in the HBII sequence that may produce changes in the protein tertiary structure. Next to the loop where we introduced the RGD sequence there is a PRARI sequence that may act as heparin-binding domain in a similar manner than the aforementioned PRRARV sequence. We postulate that there may be electric repulsion between the positive charges of the PRARI sequence and the Arg positive charge, which is the nearest amino acid in the three dimensional structure. However, further experiments should be performed to demonstrate this hypothesis.

Apart from the heparin and growth factor-binding capacity of the HBII domain, it has been observed that this fragment also participates in cell adhesion. The PRRARV 
sequence and other residues located in the $\mathrm{III}_{14}$ module, including the PRARI sequence, have also been described to regulate actin assembly and focal contacts formation. ${ }^{37,38}$ These sequences interact with cell surface proteoglycans, mainly syndecan-4, in a comparable way than heparin binding. ${ }^{23}$ Nonetheless, presence of HBII functionalized onto a surface is not enough for inducing focal adhesion formation and requires the presence of the CAS fragment from fibronectin. ${ }^{19}$ The introduction of RGD in the sequence of HBII not only preserved the syndecan-4 binding capacity of the native HBII but also stimulated the formation of focal adhesions at the spreading edges of cells, observed by both vinculin and integrin $\beta 1$ staining. The capacity of the HBII-RGD fragment to specifically bind integrin $\alpha 5 \beta 1$, the main receptor of fibronectin and the most expressed extracellular matrix receptor in undifferentiated MSCs, was demonstrated by ELISA and western blot. The novel molecule may mediate the formation of focal adhesions via two pathways. First, presence of RGD could act in synergy with syndecan-4 through protein kinase C (PKC) signalling. ${ }^{39}$ And second, physical proximity of integrins and growth factors receptors, due to the growth factor binding capacity of HBII, may facilitate their reciprocal activation controlling signalling pathways that include focal adhesion formation. ${ }^{40,41}$ During the process of cell spreading, actin polymerization drives the assembly of early cell contacts to the ECM at the leading edge of cells. ${ }^{42}$ These early contacts then maturate into focal adhesions that act as cytoskeletal organizing centers. ${ }^{43}$ Thus, presence of RGD in the novel mutated HBII fragment stimulates not only focal adhesion formation but also to some extent actin cytoskeletal assembly compared to native HBII fragment.

Finally, we wanted to know if the RGD-mutated HBII still retained its cell differentiation and mineralization capacity after the modification. The inherent growth factor binding capacity of the HBII fragment from fibronectin is responsible for 
mediating cell differentiation towards specific lineages. However, presence of CAS from fibronectin is required for the osteogenic differentiation. ${ }^{18,19}$ Herein, we demonstrated that insertion of the RGD motif in the HBII sequence improved the differentiation capacity of the native HBII fragment, as observed by increased osteogenic gene expressions. Noteworthy, mineralization, which is a potent indicator of MSCs differentiation into bone-forming cells, was also improved after the RGD mutation, being almost three times higher compared to the native HBII fragment and the CAS70-HBII30 mixture. RGD and growth factor binding sequences may act in synergy, both stimulating co-activation of integrin and growth factor receptors as explained above. It is important to highlight that osteogenic factors were not used in the present study and therefore osteogenic induction should be attributed to the binding of growth factors from the serum on the novel protein. In addition, to ensure that the novel protein binds growth factors without influencing their activity, we cultured cells in presence of BMP-2 for $2 \mathrm{~h}$. It was previously described that the HBII fragment did not influence the proliferative effect of different angiogenic factors on endothelial and smooth muscle cells. ${ }^{20}$ However, the authors did not demonstrate that HBII does not affect the activity of BMP-2. We observed that the increase of gene expressions obtained for the native HBII fragment was also noticed for the RGD-mutated fragment in presence of BMP-2. As observed in absence of BMP-2, gene expressions dramatically increased at earlier times of cell culture, a pattern also observed when cells are treated with soluble BMP2. ${ }^{44-47}$ We speculate that gene expression is induced by the attraction of BMP-2 by the native and mutated HBII fragments and therefore it is not strange to observe similar patterns compared to other BMP-2-treatment studies. Then, gene expression results demonstrate the ability of the RGD-mutated HBII functionalized Ti to retain active BMP-2 molecules thereby acting as promising osteogenic surfaces. 


\section{CONCLUSIONS}

A novel recombinant protein fragment has been generated by DNA mutation introducing an RGD motif into the HBII sequence of fibronectin. The novel protein improves cell adhesion whereas it retains cell differentiation capability when immobilized on Ti compared to the native HBII fragment, being a promising candidate to improve bone tissue implant integration. The novel strategy to produce a gain-offunction mutation into recombinant proteins opens a new window for engineering active molecules for regenerative medicine with virtually unlimited assembly design. Considering that the HBII fragment promiscuously binds several growth factors and the surprisingly high TGF- $\beta 1$ binding capacity of the novel protein, the strategy could be adapted to biomaterials for other applications. In this regard, generation of a biomaterial with controlled spatiotemporal release of growth factors is of paramount interest in contrast to the use of biomaterials doped with growth factors. Thus, integrin/growth factor receptor synergy is a key parameter for ongoing research in regenerative medicine.

\section{ASSOCIATED CONTENT}

Supporting Information. Experimental procedures for sequencing of mutated HBII; figure of DNA sequence of the mutated HBII fragment.

\section{ACKNOWLEDGEMENTS}

Authors acknowledge the financial support received from the Spanish Government through the MAT2015-67183-R project, cofunded by the EU through the European Regional Development Funds. J.G.M acknowledges his personal support through PDJ2014 fellowship from the Agency for Management of University and Research Grants (AGAUR) and M.P.G. thanks the ICREA Academia award for excellence in 
research, both funded by the Generalitat de Catalunya. Authors also thank the Generalitat de Catalunya for funding through project 2017SGR-1165.

\section{REFERENCES}

(1) Chen, Q.; Thouas, G. A. Metallic Implant Biomaterials. Mater. Sci. Eng. $R$ Reports 2015, 87, 1-57.

(2) Schliephake, H.; Scharnweber, D. Chemical and Biological Functionalization of Titanium for Dental Implants. J. Mater. Chem. 2008, 18 (21), 2404-2414.

(3) Hu, Y.; Cai, K.; Luo, Z.; Zhang, Y.; Li, L.; Lai, M.; Hou, Y.; Huang, Y.; Li, J.; Ding, X.; Zhang, B.; Sung, K.L. Regulation of the Differentiation of Mesenchymal Stem Cells in Vitro and Osteogenesis in Vivo by Microenvironmental Modification of Titanium Alloy Surfaces. Biomaterials 2012, 33 (13), 3515-3528.

(4) Kim, S. H.; Turnbull, J.; Guimond, S. Extracellular Matrix and Cell Signalling: The Dynamic Cooperation of Integrin, Proteoglycan and Growth Factor Receptor. J. Endocrinol. 2011, 209 (2), 139-151.

(5) Ghosh, K.; Ren, X.-D.; Shu, X. Z.; Prestwich, G. D.; Clark, R. A. F. Fibronectin Functional Domains Coupled to Hyaluronan Stimulate Adult Human Dermal Fibroblast Responses Critical for Wound Healing. Tissue Eng. 2006, 12 (3), 601613.

(6) Discher, D. E.; Mooney, D. J.; Zandstra, P. W. Growth Factors, Matrices, and Forces Combine and Control Stem Cells. Science 2009, 324 (5935), 1673-1677.

(7) Martino, M. M.; Briquez, P. S.; Ranga, A.; Lutolf, M. P.; Hubbell, J. A. HeparinBinding Domain of Fibrin(ogen) Binds Growth Factors and Promotes Tissue 
Repair When Incorporated within a Synthetic Matrix. Proc. Natl. Acad. Sci. 2013, $110(12), 4563-4568$.

(8) Chen, F. M.; Zhang, M.; Wu, Z. F. Toward Delivery of Multiple Growth Factors in Tissue Engineering. Biomaterials 2010, 31 (24), 6279-6308.

(9) James, A. W.; LaChaud, G.; Shen, J.; Asatrian, G.; Nguyen, V.; Zhang, X.; Ting, K.; Soo, C. A Review of the Clinical Side Effects of Bone Morphogenetic Protein-2. Tissue Eng. Part B Rev. 2016, 22 (4), 284-297.

(10) Zhu, J.; Clark, R. A. F. Fibronectin at Select Sites Binds Multiple Growth Factors and Enhances Their Activity: Expansion of the Collaborative ECM-GF Paradigm. J. Invest. Dermatol. 2014, 134 (4), 895-901.

(11) Pankov, R. Fibronectin at a Glance. J. Cell Sci. 2002, 115 (20), 3861-3863.

(12) Rivera-Chacon, D. M.; Alvarado-Velez, M.; Acevedo-Morantes, C. Y.; Singh, S. P.; Gultepe, E.; Nagesha, D.; Sridhar, S.; Ramirez-Vick, J. E. Fibronectin and Vitronectin Promote Human Fetal Osteoblast Cell Attachment and Proliferation on Nanoporous Titanium Surfaces. J. Biomed. Nanotechnol. 2013, 9 (6), $1092-$ 1097.

(13) Speziale, P.; Visai, L.; Rindi, S.; Di Poto, A. Purification of Human Plasma Fibronectin Using Immobilized Gelatin and Arg Affinity Chromatography. Nat. Protoc. 2008, 3 (3), 525-533.

(14) Franz, S.; Rammelt, S.; Scharnweber, D.; Simon, J. C. Immune Responses to Implants - A Review of the Implications for the Design of Immunomodulatory Biomaterials. Biomaterials 2011, 32 (28), 6692-6709.

(15) Petrie, T. A.; Raynor, J. E.; Dumbauld, D. W.; Lee, T. T.; Jagtap, S.; Templeman, K. L.; Collard, D. M.; García, A. J. Multivalent Integrin-Specific Ligands Enhance Tissue Healing and Biomaterial Integration. Sci. Transl. Med. 2010, 2 
(45), 45ra60.

(16) Petrie, T. A.; Reyes, C. D.; Burns, K. L.; García, A. J. Simple Application of Fibronectin-Mimetic Coating Enhances Osseointegration of Titanium Implants. J. Cell. Mol. Med. 2009, 13 (8 B), 2602-2612.

(17) Agarwal, R.; González-García, C.; Torstrick, B.; Guldberg, R. E.; SalmerónSánchez, M.; García, A. J. Simple Coating with Fibronectin Fragment Enhances Stainless Steel Screw Osseointegration in Healthy and Osteoporotic Rats. Biomaterials 2015, 63, 137-145.

(18) Martino, M. M.; Tortelli, F.; Mochizuki, M.; Traub, S.; Ben-David, D.; Kuhn, G. A.; Muller, R.; Livne, E.; Eming, S. A.; Hubbell, J. A. Engineering the Growth Factor Microenvironment with Fibronectin Domains to Promote Wound and Bone Tissue Healing. Sci. Transl. Med. 2011, 3 (100), 100 ra89.

(19) Herranz-Diez, C.; Mas-Moruno, C.; Neubauer, S.; Kessler, H.; Gil, F. J.; Pegueroles, M.; Manero, J. M.; Guillem-Marti, J. Tuning Mesenchymal Stem Cell Response onto Titanium-Niobium-Hafnium Alloy by Recombinant Fibronectin Fragments. ACS Appl. Mater. Interfaces 2016, 8 (4), 2517-2525.

(20) Martino, M. M.; Hubbell, J. A. The 12th-14th Type III Repeats of Fibronectin Function as a Highly Promiscuous Growth Factor-Binding Domain. FASEB J. 2010, 24 (12), 4711-4721.

(21) Lin, F.; Ren, X.-D.; Pan, Z.; Macri, L.; Zong, W.-X.; Tonnesen, M. G.; Rafailovich, M.; Bar-Sagi, D.; Clark, R. A. F. Fibronectin Growth FactorBinding Domains Are Required for Fibroblast Survival. J. Invest. Dermatol. 2011, 131 (1), 84-98.

(22) Elfenbein, A.; Simons, M. Syndecan-4 Signaling at a Glance. J. Cell Sci. 2013, $126(17), 3799-3804$. 
(23) Woods, A.; Longley, R. L.; Tumova, S.; Couchman, J. R. Syndecan-4 Binding to the High Affinity Heparin-Binding Domain of Fibronectin Drives Focal Adhesion Formation in Fibroblasts. Arch. Biochem. Biophys. 2000, 374 (1), 6672.

(24) Herranz-Diez, C.; Li, Q.; Lamprecht, C.; Mas-Moruno, C.; Neubauer, S.; Kessler, H.; Manero, J. M.; Guillem-Martí, J.; Selhuber-Unkel, C. Bioactive Compounds Immobilized on $\mathrm{Ti}$ and TiNbHf: AFM-Based Investigations of Biofunctionalization Efficiency and Cell Adhesion. Colloids Surf., B 2015, 136, 704-711.

(25) Guillem-Marti, J.; Delgado, L.; Godoy-Gallardo, M.; Pegueroles, M.; Herrero, M.; Gil, F. J. Fibroblast Adhesion and Activation onto Micro-Machined Titanium Surfaces. Clin. Oral Implants Res. 2013, 24 (7), 770-780.

(26) Barkalow, F. J. B.; Schwarzbauer, J. E. Localization of the Major HeparinBinding Site in Fibronectin. J. Biol. Chem. 1991, 266 (12), 7812-7818.

(27) Carpentier, M.; Denys, A.; Allain, F.; Vergoten, G. Molecular Docking of Heparin Oligosaccharides with Hep-II Heparin-Binding Domain of Fibronectin Reveals an Interplay between the Different Positions of Sulfate Groups. Glycoconj. J. 2014, 31 (2), 161-169.

(28) Cardin, A. D.; Weintraub, H. J. Molecular Modeling of ProteinGlycosaminoglycan Interactions. Arterioscler. Thromb. Vasc. Biol. 1989, 9 (1), $21-32$.

(29) Capila, I.; Linhardt, R. J. Heparin-Protein Interactions. Angew. Chem. Int. Ed. Engl. 2002, 41 (3), 391-412.

(30) Sugahara, K.; Kitagawa, H. Heparin and Heparan Sulfate Biosynthesis. IUBMB Life 2002, 54 (4), 163-175. 
(31) Martino, M. M.; Briquez, P. S.; Maruyama, K.; Hubbell, J. A. Extracellular Matrix-Inspired Growth Factor Delivery Systems for Bone Regeneration. $A d v$. Drug Deliv. Rev. 2015, 94, 41-52.

(32) Lee, K.; Silva, E. A.; Mooney, D. J. Growth Factor Delivery-Based Tissue Engineering: General Approaches and a Review of Recent Developments. J. R. Soc. Interface 2011, 8 (55), 153-170.

(33) Poon, B.; Kha, T.; Tran, S.; Dass, C. R. Bone Morphogenetic Protein-2 and Bone Therapy: Successes and Pitfalls. J. Pharm. Pharmacol. 2016, 68 (2), 139-147.

(34) Chen, G.; Deng, C.; Li, Y. P. TGF- $\beta$ and BMP Signaling in Osteoblast Differentiation and Bone Formation. Int. J. Biol. Sci. 2012, 8 (2), 272-288.

(35) Rahman, M. S.; Akhtar, N.; Jamil, H. M.; Banik, R. S.; Asaduzzaman, S. M. TGF- $\beta$ /BMP Signaling and Other Molecular Events: Regulation of Osteoblastogenesis and Bone Formation. Bone Res. 2015, 3, 15005.

(36) Wu, M.; Chen, G.; Li, Y.-P. TGF- $\beta$ and BMP Signaling in Osteoblast, Skeletal Development, and Bone Formation, Homeostasis and Disease. Bone Res. 2016, 4,16009 .

(37) Woods, A.; McCarthy, J. B.; Furcht, L. T.; Couchman, J. R. A Synthetic Peptide from the COOH-Terminal Heparin-Binding Domain of Fibronectin Promotes Focal Adhesion Formation. Mol. Biol. Cell 1993, 4 (6), 605-613.

(38) Bloom, L.; Ingham, K. C.; Hynes, R. O. Fibronectin Regulates Assembly of Actin Filaments and Focal Contacts in Cultured Cells via the Heparin-Binding Site in Repeat III13. Mol. Biol. Cell 1999, 10 (5), 1521-1536.

(39) Morgan, M. R.; Humphries, M. J.; Bass, M. D. Synergistic Control of Cell Adhesion by Integrins and Syndecans. Nat. Rev. Mol. Cell Biol. 2007, 8 (12), $957-969$. 
(40) Wei, Q.; Pohl, T. L. M.; Seckinger, A.; Spatz, J. P.; Cavalcanti-Adam, E. A. Regulation of Integrin and Growth Factor Signaling in Biomaterials for Osteodifferentiation. Beilstein J. Org. Chem. 2015, 11, 773-783.

(41) Salmerón-Sánchez, M.; Dalby, M. J. Synergistic Growth Factor Microenvironments. Chem. Commun. 2016, 52 (91), 13327-13336.

(42) Pollard, T. D.; Borisy, G. G. Cellular Motility Driven by Assembly and Disassembly of Actin Filaments. Cell 2003, 112 (4), 453-465.

(43) Borisy, G. G.; Svitkina, T. M. Actin Machinery: Pushing the Envelope. Curr. Opin. Cell Biol. 2000, 12 (1), 104-112.

(44) Lee, K. S.; Kim, H. J.; Li, Q. L.; Chi, X. Z.; Ueta, C.; Komori, T.; Wozney, J. M.; Kim, E. G.; Choi, J. Y.; Ryoo, H. M.; Bae, S. C. Runx2 Is a Common Target of Transforming Growth Factor $\beta 1$ and Bone Morphogenetic Protein 2, and Cooperation between Runx2 and Smad5 Induces Osteoblast-Specific Gene Expression in the Pluripotent Mesenchymal Precursor Cell Line C2C12. Mol. Cell. Biol. 2000, 20 (23), 8783-8792.

(45) Noël, D.; Gazit, D.; Bouquet, C.; Apparailly, F.; Bony, C.; Plence, P.; Millet, V.; Turgeman, G.; Perricaudet, M.; Sany, J.; Jorgensen, C. Short-term BMP-2 expression is sufficient for in vivo osteochondral differentiation of mesenchymal stem cells. Stem Cells 2004, 22 (1), 74-85.

(46) Balint, E.; Lapointe, D.; Drissi, H.; van der Meijden, C.; Young, D. W.; van Wijnen, A. J.; Stein, J. L.; Stein, G. S.; Lian, J. B. Phenotype discovery by gene expression profiling: Mapping of biological processes linked to BMP-2-mediated osteoblast differentiation. J. Cell. Biochem. 2003, 89 (2), 401-426.

(47) Chen, D.; Harris, M. A.; Rossini, G.; Dunstan, C. R.; Dallas, S. L.; Feng, J. Q.; Mundy, G. R.; Harris, S. E. Bone Morphogenetic Protein 2 (BMP-2) Enhances 
BMP-3, BMP-4, and Bone Cell Differentiation Marker Gene Expression During the Induction of Mineralized Bone Matrix Formation in Cultures of Fetal Rat Calvarial Osteoblasts. Calcif. Tissue Int. 1997, 60 (3), 283-290. 

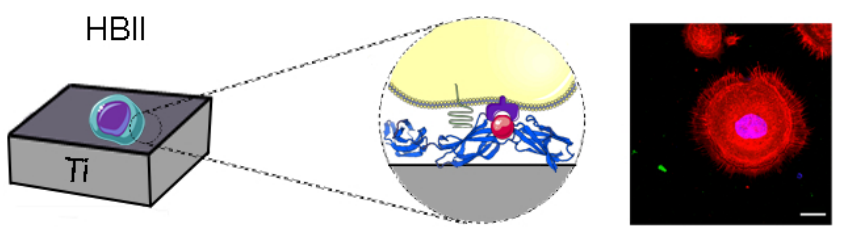

Spreading 仓 Differentiation

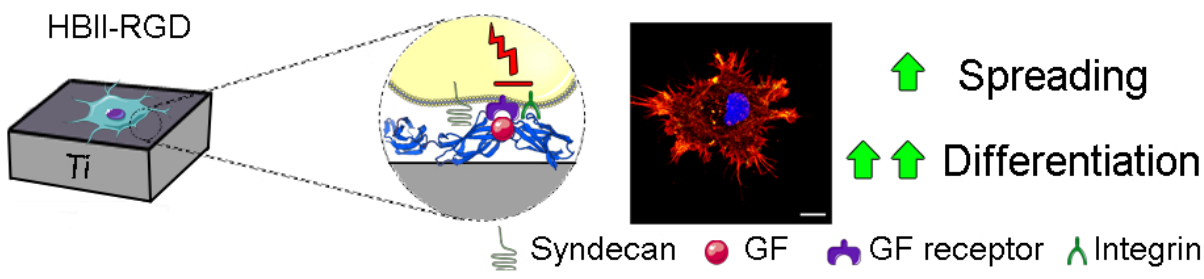

Graphical abstract

$83 \times 35 \mathrm{~mm}(300 \times 300 \mathrm{DPI})$ 